\title{
Bipartite Consensus Control of Multiagent Systems on Coopetition Networks
}

\author{
Jiangping Hu \\ School of Automation Engineering, University of Electronic Science and Technology of China, Chengdu 611731, China \\ Correspondence should be addressed to Jiangping Hu; hjp_lzu@163.com
}

Received 12 April 2014; Accepted 9 June 2014; Published 19 June 2014

Academic Editor: Norio Yoshida

Copyright (C) 2014 Jiangping Hu. This is an open access article distributed under the Creative Commons Attribution License, which permits unrestricted use, distribution, and reproduction in any medium, provided the original work is properly cited.

Cooperation and competition are two typical interactional relationships in natural and engineering networked systems. Some complex behaviors can emerge through local interactions within the networked systems. This paper focuses on the coexistence of competition and cooperation (i.e., coopetition) at the network level and, simultaneously, the collective dynamics on such coopetition networks. The coopetition network is represented by a directed signed graph. The collective dynamics on the coopetition network is described by a multiagent system. We investigate two bipartite consensus strategies for multiagent systems such that all the agents converge to a final state characterized by identical modulus but opposite sign. Under a weak connectivity assumption that the coopetition network has a spanning tree, some sufficient conditions are derived for bipartite consensus of multiagent systems with the help of a structural balance theory. Finally, simulation results are provided to demonstrate the bipartite consensus formation.

\section{Introduction}

Complex systems research is becoming ever more important in both the natural and social sciences, even though there is no formal definition of complex systems under a uniforming framework for science. Complex systems generally involve with many autonomous components, local interactions, emergent behaviors, and so on $[1,2]$. The interactions or relationship between components or agents can be diverse, for example, cooperation or competition. Different behaviors, such as synchronization or consensus, polarization, and fragmentation, can emerge through local interactions between agents $[3,4]$.

Till now, there has been a surge of attention paid to the study of multiagent systems where agents interact cooperatively. A typical collective behavior is characterized by the emergence of a global consensus, in which all agents reach the same state in the long run [5]. Some pioneering physical models were proposed to investigate various consensus behaviors, for example, fireflies' flashing [6], opinion agreement [7], clapping synchronization [8], phase transition of self-driven particles [9], and oscillator synchronization [10]. During the last few years, large numbers of theoretical results have been obtained for consensus behaviors under the framework of multiagent systems and complex networks [1114]. In the study of consensus, a graph is normally used to model multiagent systems, with nodes representing agents and (positive) edges describing their pairwise cooperation. It has been shown that global consensus can be reached if and only if directed graphs associated with multiagent systems have a spanning tree [15-18].

In many real-world scenarios, another type of "consensus" phenomenon has been observed for a long time, where all agents reach a final state with identical magnitude but opposite sign. Hereafter, we call such kind of collective behavior bipartite consensus or antisynchronization. For example, a polarization often happens in a two-coalition community such that opposite opinions are held by two fractions $[19,20]$. Antisynchronization is a noticeable phenomenon in periodic oscillators, which has been investigated for Lorenz or Chua's chaotic systems [21, 22]. In statistics physics field, a classical model, Ising model, was built to describe the crystal magnetization phenomenon, where the agents (electrons) interact through ferromagnetic or antiferromagnetic interactions and antisynchronization can be emerged under some critical condition [23]. In order to study bipartite consensus, the 
interaction networks among agents are generally modeled by signed graphs with positive/negative edges [24]. Furthermore, the evolution of the collective dynamics is generally analyzed by using the notion of structural balance [25-27]. Structural balance is an important property in social network theory, which partitions signed graphs into two subgraphs such that each subgraph contains only positive edges while all edges joining different subgraphs are negative $[24,28]$.

Closely related with this paper is the bipartite consensus formation on coopetition networks, which was initially discussed in [26]. A sufficient and necessary condition was presented to ensure that bipartite consensus can be reached if and only if the signed graphs associated with multiagent systems are strongly connected and structurally balanced. Some sufficient conditions have been given in [27] to ensure that bipartite consensus can be reached for agents over a directed signed network with a spanning tree. In this paper, a complete analysis is provided for bipartite consensus formation for multiagent systems on interaction networks containing relationships of cooperation and competition, which are called coopetition networks for convenience and modeled by a directed signed graph. The coopetition network is assumed to have a spanning tree, which means that there is at least one agent, from which the other agents can receive the information directly or indirectly, in the multiagent systems. We consider two cases. (i) One is that all agents exchange their states cooperatively or competitively without any exogenous influence or hint. (ii) The other is that a visible or invisible leader exists and influences the remainder. Then two questions arise. Can the agents realize a bipartite consensus with/without exogenous influence? Is there difference between the evolutions of multiagent systems under the two cases? In order to answer the questions, we propose a free bipartite consensus control and an interventional bipartite consensus control for multiagent systems and investigate the bipartite consensus formation under the two strategies. Thus, the contributions of this paper are devoted to deriving some sufficient conditions related to structural balance for bipartite consensus on coopetition networks and demonstrate the bipartite consensus formation for a complete category of coopetition networks.

The remainder of this paper is organized as follows. In Section 2, a bipartite consensus problem is formulated and coopetition networks are modeled appropriately. In Section 3, two bipartite consensus strategies of multiagent systems on coopetition networks are proposed and the convergence analysis of the bipartite consensus formation is presented. Simultaneously, some sufficient conditions are obtained to describe cases in which bipartite consensus can be achieved. In Section 4, some simulations are provided to demonstrate the bipartite consensus formation of multiagent systems with/without exogenous information. Finally, we draw a conclusion in Section 5.

\section{Problem Formulation}

2.1. Coopetition Network Modeling. When we regard an agent as a node and the interactions between two agents as directed edges, it is helpful to use directed signed graphs to describe coopetition networks. The positive and negative edges in directed signed graphs represent, respectively, the cooperative and competitive interactions in coopetition networks.

Formally, a directed signed graph is a directed graph $\mathscr{G}^{s}=$ $\{\mathscr{V}, \mathscr{E}, A\}$, where $\mathscr{V}=\{1, \ldots, n\}$ is a set of nodes, $\mathscr{E} \subseteq \mathscr{V} \times \mathscr{V}$ is a set of edges, and $A$ is an adjacency matrix describing the edge information of a positive or negative sign. The nonzero element $a_{i j}$ of $A$ is attached to the edge $(j, i) \in \mathscr{E}$, which is directed from node $j$ to node $i$. In this way, the node $j$ is called the parent node and $i$ is the child node, which means that the information flow starts from agent $j$ and ends at agent $i$. The edge set $\mathscr{E}=\mathscr{E}^{+} \cup \mathscr{E}^{-}$, where $\mathscr{E}^{+}=$ $\left\{(j, i) \mid a_{i j}>0\right\}$ and $\mathscr{E}^{-}=\left\{(j, i) \mid a_{i j}<0\right\}$ are the sets of positive and negative edges, respectively. If all edges are positive and $\mathscr{E}^{-}=\emptyset$, the graph is simply a directed unsigned graph or digraph $\mathscr{G}$. A directed path is a sequence of edges of the form $\left(i_{1}, i_{2}\right),\left(i_{2}, i_{3}\right), \ldots,\left(i_{l-1}, i_{l}\right)$ with distinct nodes with length $l-1$. A semipath is defined as a sequence of nodes $i_{1}, \ldots, i_{l}$ such that either $\left(i_{\kappa}, i_{\kappa-1}\right)$ or $\left(i_{\kappa-1}, i_{\kappa}\right)$ belongs to the set $\mathscr{E}$. A directed (semi)cycle is a directed (semi)path beginning and ending with the same nodes. A directed graph $\mathscr{G}^{s}$ is said to be strongly (weakly) connected if there is a directed path (semipath) between any pair of distinct nodes. A directed tree is a directed graph, where every node, except the root, has exactly one parent. A spanning tree of a directed graph is a directed tree containing directed paths from the root to all other nodes of the graph. Herein, we give an intuitive illustration of coopetition networks in Figure 1.

In Figures 1(a) and 1(b), the two networks $\mathscr{G}^{s}$ and $\mathscr{G}_{\text {Tree }}^{s}$ are signed graphs, which have positive and negative edges. The positive and negative edges are denoted by blue solid and red dash lines, respectively. The graph $\mathscr{G}_{\text {Tree }}^{s}$ is a spanning tree of $\mathscr{G}^{s}$ and the node 1 is a root node. The network $\mathscr{G}^{s}$ has two subnetworks $A$ and $B$. The edges are positive within each subnetwork and are negative between the two subnetworks. In Figure 1(a), the nodes 1, 7, 8, 9, and 2 form a semicycle. In Figure 1(b), the node 1 has no parent node and all the other nodes have only one parent node; therefore, $\mathscr{G}_{\text {Tree }}^{s}$ is a spanning tree of $\mathscr{G}^{s}$. A coopetition network having a spanning tree means that there exists at least one root agent who may not be influenced by any other agents, and at the same time, the remaining nodes are influenced directly or indirectly by the root agent.

A (semi)cycle in coopetition networks generally contains cooperative and competitive interactions. If the product of the weights $a_{i j}$ in the (semi)cycle is positive, then we say that the (semi)cycle is positive, and negative, otherwise. Obviously, the semicycle $1 \rightarrow 7 \rightarrow 8 \rightarrow 9 \rightarrow 2 \rightarrow$ 1 is positive since there are two negative edges $1 \leftrightarrow 7$ and $2 \rightarrow 9$ in $\mathscr{G}^{s}$ in Figure 1(a). A coopetition network $\mathscr{G}^{s}$ is said structurally balanced if all of its semicycles are positive [24], and $\mathscr{G}^{s}$ is said structurally unbalanced if one of its semicycles is negative. It is noticed that the existence of semicycles is a necessary condition of structural balance. If a network $\mathscr{G}^{s}$ has no semicycles, it is said vacuously balanced [28]. A coopetition network is said to be homogeneous if all the interactions are cooperative or if all the interactions are competitive, and heterogeneous otherwise. Additionally, 


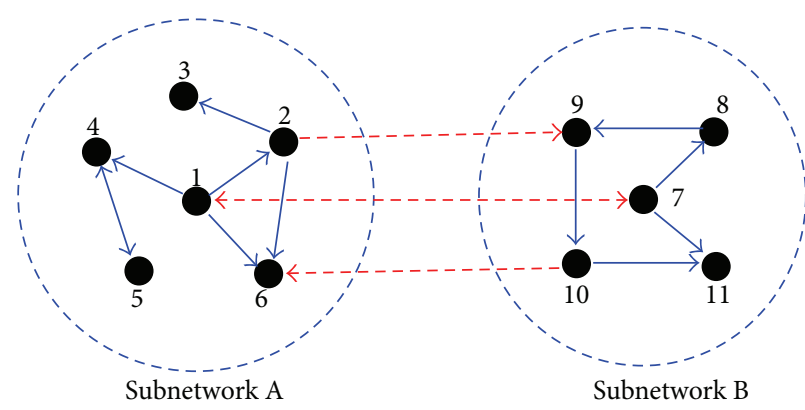

(a) $\mathscr{G}^{s}$

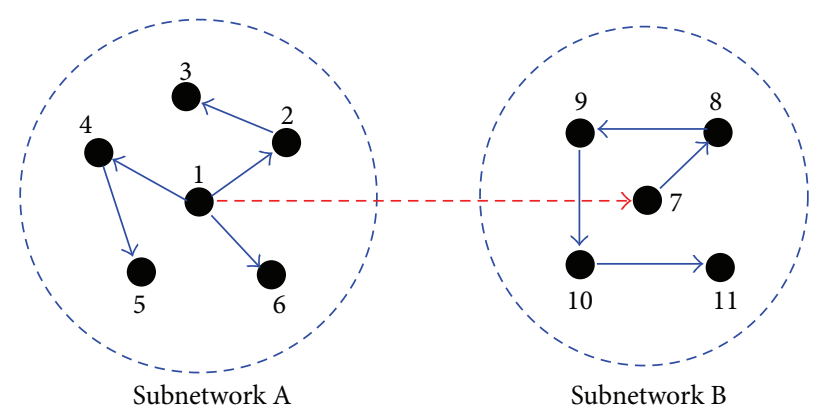

(b) $\mathscr{G}_{\text {Tree }}^{s}$

FIgURE 1: Coopetition networks.

homogeneous coopetition networks have two classes: allpositive networks (i.e., all of the edges are positive) and allnegative networks (i.e., all of the edges are negative).

Notice that there is a common phenomenon that a structurally balanced coopetition network generally consists of two subnetworks. The interactions are cooperative within each subnetwork, but competitive between the two subnetworks. Additionally, a coopetition network is called bipartite network if it can be partitioned into two subnetworks such that all interactions only exist between the different subnetworks. Obviously, in a bipartite coopetition network, agents have no relationship even though they are belonging to the same subnetwork. The two subnetworks may cooperate or compete.

2.2. Bipartite Consensus Problem. Consider that a group of agents, labeled by $1, \ldots, n$, interact cooperatively or competitively on a network described by a signed graph $\mathscr{G}^{s}$. The dynamics of agent $i$ is expressed by a first-order integrator

$$
\dot{x}_{i}(t)=u_{i},
$$

where $x_{i}(t) \in \mathbb{R}$ is the state and $u_{i}(t) \in \mathbb{R}$ is the control strategy of agent $i$.

In the bipartite consensus problem under investigation, we restrict our attention to analyzing how the structurally balanced condition plays a key role in the bipartite consensus formation. More specifically, structural conditions are explored for a complete category of coopetition networks such that all agents reach bipartite consensus; that is,

$$
\lim _{t \rightarrow \infty}\left|x_{i}(t)\right|=\mathbf{c}>0,
$$

for all $i=1, \ldots, n$. The consensus state $\mathbf{c}$ generally depends on the initial state of the multiagent system (1). For convenience, the bipartite consensus without exogenous influence is called free bipartite consensus. Specifically, if the interaction network $\mathscr{G}^{s}$ associated with the multiagent system (1) is structurally balanced or bipartite, $\mathscr{G}^{s}$ can be divided into two subnetworks with node sets $\mathscr{V}_{1}=\{1, \ldots, m\} \quad(1 \leq m<n)$ and $\mathscr{V}_{2}=\{m+1, \ldots, n\}$, respectively, and then the free bipartite consensus means that, for each agent belonging to subgroup $\mathscr{V}_{1}$ or $\mathscr{V}_{2}$,

$$
\lim _{t \rightarrow \infty} x_{i}(t)=\mathbf{c},
$$

for $i \in \mathscr{V}_{1}$, while

$$
\lim _{t \rightarrow \infty} x_{i}(t)=-\mathbf{c},
$$

for $i \in \mathscr{V}_{2}$. Additionally, if the consensus state is given by

$$
\mathbf{c}=\frac{1}{n}\left|\omega^{T} x(0)\right|
$$

for some constant weight vector $\omega$, then we say that all the agents reach an average bipartite consensus. Furthermore, if the final consensus state $\mathbf{c}$ can be steered according to some given policy or exogenous information, a virtual leader has to be introduced to coordinate two parts of agents as an intercessor. Thus, the consensus state $\mathbf{c}$ is completely determined by the virtual leader, which is called an interventional bipartite consensus. Additionally, the interaction network involving the virtual leader is described by another coopetition network $\overline{\mathscr{G}}^{s}=\{\overline{\mathscr{V}}, \overline{\mathscr{C}}\}$ with $\overline{\mathscr{V}}=\overline{\mathscr{V}} \cup\{0\}$ and $\overline{\mathscr{E}} \subseteq \overline{\mathscr{V}} \times \overline{\mathscr{V}}$. Assume that the dynamics of the virtual leader is described by a first-order exogenous system as follows:

$$
\dot{x}_{0}(t)=u_{0}(t),
$$

where $x_{0}(t) \in \mathbb{R}$ and $u_{0}(t) \in \mathbb{R}$ denote the state and the input of the exogenous system. The input $u_{0}(t)$ of the exogenous system (6) is a common policy and is assumed to be known by all the agents. Then the interventional bipartite consensus problem is mainly devoted to designing appropriate coordination strategies for the coopetition agents such that both the two competitive parts can reach the state of the virtual leader; that is, for the agents belonging to the parts $\mathscr{V}_{1}$ and $\mathscr{V}_{2}$,

$$
\lim _{t \rightarrow \infty}\left[x_{i}(t)-x_{0}(t)\right]=0,
$$

for $i \in \mathscr{V}_{1}$, while

$$
\lim _{t \rightarrow \infty}\left[x_{i}(t)+x_{0}(t)\right]=0,
$$

for $i \in \mathscr{V}_{2}$.

In the next section, two bipartite consensus strategies are designed for each agent on the coopetition network $\mathscr{G}^{s}$ to realize the free bipartite consensus or the interventional bipartite consensus. Then, the collective dynamics with the proposed bipartite consensus strategy will be analyzed for homogeneous and heterogeneous coopetition networks, respectively. 


\section{Main Results}

3.1. Free Bipartite Consensus. In order to realize the free bipartite consensus, a coordination strategy is firstly proposed for the two groups of the coopetition agents. Suppose the coopetition network $\mathscr{G}^{s}$ is divided into two subnetworks $\mathscr{G}_{1}^{s}$ and $\mathscr{G}_{2}^{s}$, which have two vertex sets $\mathscr{V}_{1}=\{1, \ldots, m\} \quad(1 \leq$ $m<n)$ and $\mathscr{V}_{2}=\{m+1, \ldots, n\}$, respectively. Then, the coordination strategy is proposed for agent $i$ in the subnetwork $\mathscr{G}_{1}^{s}$,

$$
u_{i}(t)=\sum_{j \in \mathcal{N}_{i}^{1}} a_{i j}\left[x_{j}(t)-x_{i}(t)\right]+\sum_{j \in \mathcal{N}_{i}^{2}} a_{i j}\left[x_{j}(t)+x_{i}(t)\right],
$$

while for the opponents in the subnetwork $\mathscr{G}_{2}^{s}$,

$$
u_{i}(t)=\sum_{j \in \mathcal{N}_{i}^{2}} a_{i j}\left[x_{j}(t)-x_{i}(t)\right]+\sum_{j \in \mathcal{N}_{i}^{1}} a_{i j}\left[x_{j}(t)+x_{i}(t)\right],
$$

where $\mathscr{N}_{i}^{1}$ and $\mathscr{N}_{i}^{2}$ denote, respectively, the neighbor set belonging to the two subnetworks $\mathscr{G}_{1}^{s}$ and $\mathscr{G}_{2}^{s}$ for agent $i$; that is, $\mathcal{N}_{i}^{1}=\left\{j \in \mathscr{V}_{1} \mid a_{i j} \neq 0\right\}, \mathcal{N}_{i}^{2}=\left\{j \in \mathscr{V}_{2} \mid a_{i j} \neq 0\right\}$.

The control (9a)-(9b) can be simplified as

$$
u_{i}(t)=\sum_{j \in \mathcal{N}_{i}^{1} \cup \mathcal{N}_{i}^{2}} a_{i j}\left[x_{j}(t)-\operatorname{sgn}\left(a_{i j}\right) x_{i}(t)\right] .
$$

Applying the strategy (10) to the agent dynamics (1) leads to

$$
\dot{x}_{i}(t)=\sum_{j \in \mathcal{N}_{i}^{1} \cup \mathcal{N}_{i}^{2}} a_{i j}\left[x_{j}(t)-\operatorname{sgn}\left(a_{i j}\right) x_{i}(t)\right],
$$

which can be written in a matrix form

$$
\dot{x}(t)=-L^{s} x(t),
$$

where $x(t)=\operatorname{col}\left(x_{1}(t), \ldots, x_{n}(t)\right) \in \mathbb{R}^{n}$. The state matrix $L^{s} \in$ $\mathbb{R}^{n \times n}$ is called a signed Laplacian matrix of a directed signed graph $\mathscr{G}^{s}$ and is defined as

$$
L^{s}=C_{r}-A
$$

where $A$ is the adjacency matrix and $C_{r}$ is a diagonal matrix with diagonal elements

$$
c_{r, i j}= \begin{cases}\sum_{j \in \mathcal{N}_{i}}\left|a_{i j}\right|, & i=j, \\ 0, & i \neq j .\end{cases}
$$

It is remarkably noted that the adjacency matrix $A$ is no more a nonnegative matrix and the signed Laplacian matrix $L^{s}$ generally is not a symmetric matrix as well. Particularly, if the coopetition network $\mathscr{G}^{s}$ is all-negative, the signed Laplacian matrix $L^{s}$ is called a signless matrix and denoted by $L^{-}$. The signed Laplacian matrix has some distinct differences in the spectral properties compared with a Laplacian matrix associated with a directed graph. For example, the all-ones vector $\mathbf{1}=\operatorname{col}(1, \ldots, 1)$ generally is not a zero-eigenvector of the signed Laplacian matrix. Specifically speaking, the spectral property of the signed Laplacian matrix is described by Lemma 1 .
Lemma 1. If one of the following two assumptions holds, (i) a homogeneous coopetition network is bipartite and has a spanning tree; (ii) a heterogeneous coopetition network $\mathscr{G}^{s}$ has a spanning tree and is structurally balanced, then all eigenvalues of the signed Laplacian matrix $L^{s}$ have nonnegative real-parts and 0 is a simple eigenvalue.

Proof. If the coopetition network $\mathscr{G}^{s}$ is homogeneous and allpositive, the signed Laplacian matrix returns to a Laplacian matrix. It is well known that all the eigenvalues of a Laplacian matrix have nonnegative real-parts and 0 is a simple eigenvalue if the associated digraph has a spanning tree.

If $\mathscr{G}^{s}$ is all-negative and is bipartite, no matter whether it is structurally balanced or vacuously balanced, then all the nodes in $\mathscr{G}^{-}$can be partitioned into two subsets $\mathscr{V}_{1}$ and $\mathscr{V}_{2}$ such that

$$
\begin{gathered}
\mathscr{V}=\mathscr{V}_{1} \bigcup \mathscr{V}_{2}, \quad \mathscr{V}_{1} \bigcap \mathscr{V}_{2}=\varnothing, \\
a_{i j}=0, \quad \forall i, j \in \mathscr{V}_{q}(q=1,2), \\
a_{i j} \leq 0, \quad \forall i \in \mathscr{V}_{q}, \quad j \in \mathscr{V}_{r}, q \neq r .
\end{gathered}
$$

Define a diagonal matrix

$$
\Phi=\operatorname{diag}\left\{\phi_{1}, \ldots, \phi_{n}\right\} \in \mathbb{R}^{n \times n},
$$

where $\phi_{i}=1$ when node $i$ belongs to $\mathscr{V}_{1}$ and $\phi_{i}=-1$ when node $i$ belongs to $\mathscr{V}_{2}$ and $\operatorname{diag}\{\cdot\}$ denotes a diagonal matrix by placing a vector along the diagonal direction. Obviously, the inverse matrix of $\Phi$ is itself; that is,

$$
\Phi^{2}=I \text {. }
$$

The adjacency matrix $A$ of the all-negative signed graph $G^{-}$ can be rearranged in the following form:

$$
A=\left(\begin{array}{cc}
0 & A_{12} \\
A_{21} & 0
\end{array}\right)
$$

where $A_{12}$ and $A_{21}$ are nonpositive submatrices. Multiplying by $\Phi$ on the left and right sides of $A$ yields

$$
\Phi A \Phi=\left(\begin{array}{cc}
0 & -A_{12} \\
-A_{21} & 0
\end{array}\right)
$$

which becomes a nonnegative adjacency matrix. Then, for the signless Laplacian matrix, one has

$$
\Phi L^{-} \Phi=C_{r}-\Phi A \Phi
$$

which is a Laplacian matrix associated with a directed graph $\mathscr{G}$. When $\mathscr{G}$ has a spanning tree, $\Phi L^{-} \Phi$ has a simple eigenvalue 0 . Since $L^{-}$is similar to $\Phi L^{-} \Phi$, the conclusion follows.

If the coopetition network $\mathscr{G}^{s}$ is heterogeneous, although $\mathscr{G}^{s}$ is structurally balanced, it can be partitioned into two subgraphs with node sets $\mathscr{V}_{i}(i=1,2)$, and then the adjacency matrix $A$ can be rewritten in a block form as

$$
A=\left(\begin{array}{ll}
A_{11} & A_{12} \\
A_{21} & A_{22}
\end{array}\right),
$$


where $A_{i i}(i=1,2)$ are nonnegative submatrices associated with edges within the two subgraphs while $A_{i j}(i \neq j)$ are nonpositive submatrices associated with edges between the two subgraphs. Given a transformation matrix $\Phi$ defined in (16), it is not difficult to show that

$$
\Phi L^{s} \Phi=C_{r}-\Phi A \Phi
$$

is a Laplacian matrix of $\mathscr{G}$. Thus, the conclusion follows as well.

With the help of Lemma 1, one has the following main result.

Theorem 2. If the interaction network $\mathscr{G}^{s}$ satisfies one of the following two assumptions: (i) $\mathscr{G}^{s}$ is homogeneous, bipartite and has a spanning tree; (ii) $\mathscr{G}^{s}$ is heterogeneous, structurally balanced and has a spanning tree, then all the agents reach the free bipartite consensus. Furthermore, if $\mathscr{G}^{s}$ is weight balanced, then an average bipartite consensus will be achieved.

Proof. If the interaction network $\mathscr{G}^{s}$ is bipartite or structurally balanced, then there exists a transformation matrix $\Phi$ defined by (16). For the system (12), define $\tilde{x}(t)=\Phi x(t)$. One has

$$
\dot{\tilde{x}}(t)=-L_{\Phi} \tilde{x}(t),
$$

where

$$
L_{\Phi}=\Phi L^{s} \Phi
$$

is called a transformed signed Laplacian matrix. According to Lemma 1, the transformed signed Laplacian matrix $L_{\Phi}$ is a Laplacian matrix and 0 is its simple eigenvalue; moreover, the vector $\mathbf{1}=\operatorname{col}(1, \ldots, 1)$ is the corresponding right eigenvector. Let $\alpha_{\Phi}$ be a left eigenvector of the eigenvalue 0 and satisfy $\alpha_{\Phi}^{T} \mathbf{1}=1$. We can always find a nonsingular matrix $U$ such that

$$
U^{-1} L_{\Phi} U=J_{\Phi}
$$

where $J_{\Phi}$ is the Jordan matrix associated with $L_{\Phi}$. Furthermore, one has

$$
\lim _{t \rightarrow \infty} e^{-L_{\Phi} t}=\lim _{t \rightarrow \infty} U e^{-J_{\Phi} t} U^{-1}=Q_{\Phi}=1 \alpha_{\Phi}^{T}
$$

where $Q_{\Phi}$ has only one nonzero element satisfying $q_{\Phi, 11}=1$. Then we obtain that

$$
\lim _{t \rightarrow \infty} \tilde{x}(t)=\alpha_{\Phi}^{T} \tilde{x}(0) 1
$$

or

$$
\lim _{t \rightarrow \infty} x(t)=\alpha_{\Phi}^{T} \Phi x(0) \Phi \mathbf{1}
$$

Therefore, the two competitive groups of agents reach the free bipartite consensus; that is,

$$
x_{i}(t) \longrightarrow \alpha_{\Phi}^{T} \Phi x(0)
$$

for $i \in \mathscr{V}_{1}$, while

$$
x_{i}(t) \longrightarrow-\alpha_{\Phi}^{T} \Phi x(0)
$$

for $i \in \mathscr{V}_{2}$.

Furthermore, if the coopetition network $\mathscr{G}^{s}$ is also weight balanced, then the left eigenvector of $L_{\Phi}$ is

$$
\alpha_{\Phi}=\frac{1}{n} \mathbf{1}
$$

and all the agents will reach an average bipartite consensus; that is,

$$
\lim _{t \rightarrow \infty} x(t)=\frac{1}{n} \mathbf{1}^{T} \Phi x(0) \Phi \mathbf{1} .
$$

Thus, the conclusions of the theorem follow.

Remark 3. When a coopetition network $\mathscr{G}^{s}$ is homogeneous and all-positive, that is, all agents interact cooperatively, then all agents will reach consensus; that is, $x_{i}(t) \rightarrow \mathrm{c}$ as $t \rightarrow \infty$, under the assumption that the network has a spanning tree [15].

Remark 4 (in [29]). A master stability function method was proposed to analyze the synchronization stability for a group of identical oscillators. The method firstly supposed that there exists a synchronization state for the coupled system and then got a series of decoupled variation equations for the coupled system through linearization at the synchronization state and calculated the maximal Lyapunov exponent to determine whether the synchronization state is stable with the coupling strength. In this paper, we essentially consider a partial synchronization problem for two groups of agents on coopetition networks. The equilibrium of the coupled system cannot be known beforehand and its stability mainly relies on the spectral analysis (i.e., determining the properties of the eigenvalues and the associated eigenvectors) of the signed Laplacian matrices of coopetition networks.

3.2. Interventional Bipartite Consensus. In Section 3.1, when all the agents on the coopetition network $\mathscr{G}^{s}$ can reach the free bipartite consensus, the moduli of their final states converge to a value $c$, which depends on the initial states of all the agents. However, in many cases, the final state $c$ has to be decided by an exogenous policy. Thus, in order to realize the interventional bipartite consensus, we assume that the interaction network can be divided into two subnetworks $\mathscr{G}_{1}^{s}$ and $\mathscr{G}_{2}^{s}$ and propose a new coordination strategy as follows:

$$
\begin{array}{r}
u_{i}(t)=\sum_{j \in \mathcal{N}_{i}^{1} \cup \mathcal{N}_{i}^{2}} a_{i j}\left[x_{j}(t)-\operatorname{sgn}\left(a_{i j}\right) x_{i}(t)\right] \\
+b_{i}\left[x_{0}(t)-x_{i}(t)\right]+u_{0}(t),
\end{array}
$$

for $i \in \mathscr{V}_{1}$, and

$$
\begin{array}{r}
u_{i}(t)=\sum_{j \in \mathcal{N}_{i}^{1} \cup \mathcal{N}_{i}^{2}} a_{i j}\left[x_{j}(t)-\operatorname{sgn}\left(a_{i j}\right) x_{i}(t)\right] \\
+b_{i}\left[-x_{0}(t)-x_{i}(t)\right]-u_{0}(t),
\end{array}
$$


for $i \in \mathscr{V}_{2}$. The weights $b_{i}$ 's describe the interaction relationships between the agents $i=1, \ldots, n$ and the virtual leader; that is, $b_{i}>0$ if and only if there exists an information flow starting from the leader to the agent $i$.

The leader intervention strategy (33a)-(33b) can be further reduced to

$$
\begin{gathered}
u_{i}(t)=\sum_{j \in \bigcup \mathcal{N}_{i}^{2}} a_{i j}\left[x_{j}(t)-\operatorname{sgn}\left(a_{i j}\right) x_{i}(t)\right] \\
+b_{i}\left[\operatorname{sgn}\left(\phi_{i}\right) x_{0}(t)-x_{i}(t)\right] \\
+\operatorname{sgn}\left(\phi_{i}\right) u_{0}(t),
\end{gathered}
$$

where $\phi_{i}$ 's are the diagonal elements of the matrix defined by (16).

The agent dynamics (1) is exerted by the leader intervention strategy (34) and transformed to a compact form as follows:

$$
\dot{x}(t)=-\left(L^{s}+B\right) x(t)+B \Phi \mathbf{1} x_{0}(t)+\Phi \mathbf{1} u_{0}(t),
$$

where $B=\operatorname{diag}\left\{b_{1}, \ldots, b_{n}\right\}$ is a diagonal matrix.

Lemma 5. If the coopetition network $\mathscr{G}^{\text {s }}$ satisfies one of the following two assumptions: (i) $\mathscr{G}^{s}$ is homogeneous and bipartite; (ii) $\mathscr{G}^{s}$ is heterogeneous and structurally balanced, furthermore, the leader is a root node of the coopetition network $\overline{\mathscr{G}}^{s}$, then the matrix $H^{s}=L^{s}+B$ is positive stable (i.e., $-H^{s}$ is a stable matrix).

Proof. If the coopetition network $\mathscr{G}^{s}$ satisfies one of the two assumptions mentioned above, there exists a diagonal matrix $\Phi$ defined by (16), which has the property that $\Phi^{2}=I$. As has been shown in Lemma $1, \Phi L^{s} \Phi$ is a Laplacian matrix of a digraph $\mathscr{G}$. Additionally, since the matrix $B$ is also a diagonal matrix, one has $\Phi B \Phi=B$. Thus, $\Phi H^{s} \Phi=\Phi L^{s} \Phi+B$. According to Lemma 4 described in [17], we know that the matrix $\Phi H^{s} \Phi$ is positive stable if the leader is a root node of the coopetition network $\overline{\mathscr{G}}^{s}$. Thus the conclusion follows.

Now a main result is presented for the interventional bipartite consensus problem.

Theorem 6. If the coopetition network $\mathscr{G}^{s}$ satisfies one of the following two assumptions: (i) $\mathscr{G}^{s}$ is homogeneous and bipartite; (ii) $\mathscr{G}^{s}$ is heterogeneous and structurally balanced, furthermore, the leader is a root node of the coopetition network $\overline{\mathscr{G}}^{s}$, then all the agents reach the interventional bipartite consensus.

Proof. If the interaction network $\mathscr{G}^{s}$ is bipartite or structurally balanced, there exists a transformation matrix $\Phi=$ $\operatorname{diag}\left\{\phi_{1}, \ldots, \phi_{n}\right\}$. For the system (35), define $\tilde{x}(t)=\Phi x(t)$. One has

$$
\begin{aligned}
\dot{\tilde{x}}(t) & =-\Phi H^{s} \Phi \tilde{x}(t)+\Phi B \Phi \mathbf{1} x_{0}(t)+\Phi^{2} \mathbf{1} u_{0}(t) \\
& =-\Phi H^{s} \Phi \tilde{x}(t)+B \mathbf{1} x_{0}(t)+\mathbf{1} u_{0}(t)
\end{aligned}
$$

due to the fact that $\Phi^{2}=I$.

$$
\begin{aligned}
& \text { Let } \bar{x}(t)=\tilde{x}(t)-\mathbf{1} x_{0}(t) \text { and } H=\Phi H^{s} \Phi \text {. Then one has } \\
& \qquad \dot{\bar{x}}(t)=-H \bar{x}(t)-H \mathbf{1} x_{0}(t)+B \mathbf{1} x_{0}(t)
\end{aligned}
$$

Since $\Phi L^{s} \Phi \mathbf{1}=0$ according to Lemma 5; therefore, $H \mathbf{1}=B \mathbf{1}$. Thus, one has

$$
\dot{\bar{x}}(t)=-H \bar{x}(t)
$$

From Lemma 5, $H=\Phi H^{s} \Phi$ is positive stable; therefore, the system (38) is asymptotically stable; that is, $\lim _{t \rightarrow \infty} \bar{x}(t)=$ 0 . Then, it is easy to obtain that, as $t \rightarrow \infty, \Phi x(t)-x_{0}(t) \rightarrow$ 0 , which is equivalent to the following two equations:

$$
x_{i}(t)-x_{0}(t) \longrightarrow 0,
$$

for $i \in \mathscr{V}_{1}$, and

$$
x_{i}(t)+x_{0}(t) \longrightarrow 0
$$

for $i \in \mathscr{V}_{2}$. Consequently, all the agents reach the interventional bipartite consensus. The proof is completed.

Corollary 7. If all agents interact cooperatively and the coopetition network $\overline{\mathscr{G}}^{s}$ is homogeneous and all-positive, then all agents will realize a consensus tracking; that is, $x_{i}(t)-x_{0}(t) \rightarrow$ 0 as $t \rightarrow \infty$, under the assumption that the virtual leader is a root node of $\overline{\mathscr{G}}^{s}$.

\section{Simulation Results}

In this section, we provide some simulations to demonstrate the bipartite consensus of the multiagent system (1) with the free bipartite consensus control (10) and the interventional bipartite consensus control (34), respectively. The coopetition network $\mathscr{G}^{s}$ is heterogeneous in Examples 8 and 10 and homogeneous in Example 9. As mentioned in Remark 3 and Corollary 7, when the interaction network is all-positive, bipartite consensus cannot be reached for agents and thus the multiagent system (1) on such cooperative networks is not considered in this section.

Example 8. Consider that a group of agents interact on the coopetition network $\mathscr{G}^{s}$, as illustrated in Figure 1(a). It has been known that $\mathscr{G}^{s}$ is structurally balanced and can be divided into two competitive subgroups $\mathscr{V}_{1}=\{1,2,3,4,5,6\}$ and $\mathscr{V}_{2}=\{7,8,9,10,11\}$. The coopetition network $\mathscr{G}^{s}$ has a spanning tree, where the agent 1 is a root node of the coopetition network. Suppose that the initial collective state is given by $x(0)=\operatorname{col}(1,-3,5,2,-1,4,-2,-1,3,1,-1)$ and the matrix $A$ is a normal adjacency matrix of the coopetition network $\mathscr{G}^{s}$; that is, $a_{i j}=0,1$ or -1 . It is not difficult to know that the eigenvalues of the signed Laplacian matrix $L^{s}$ are, respectively, $1,3,2,1,2,1,1,2.618,2,0.382,0$ and 0 is a simple eigenvalue, which validates Lemma 1.

The states of the two competitive subgroups evolve on the coopetition network $\mathscr{G}^{s}$, which is illustrated in Figure 2. In Figure 2, the solid and dash edges, respectively, denote the state evolutions of the agents belonging to $\mathscr{V}_{1}$ and $\mathscr{V}_{2}$. Since the coopetition network $\mathscr{G}^{s}$ satisfies the sufficient conditions 


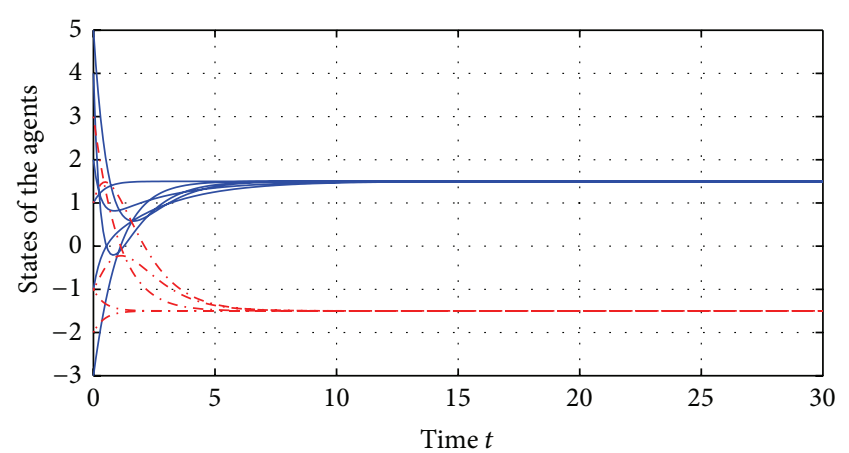

FIGURE 2: State evolution of agents on the structurally balanced coopetition network $\mathscr{G}^{s}$.

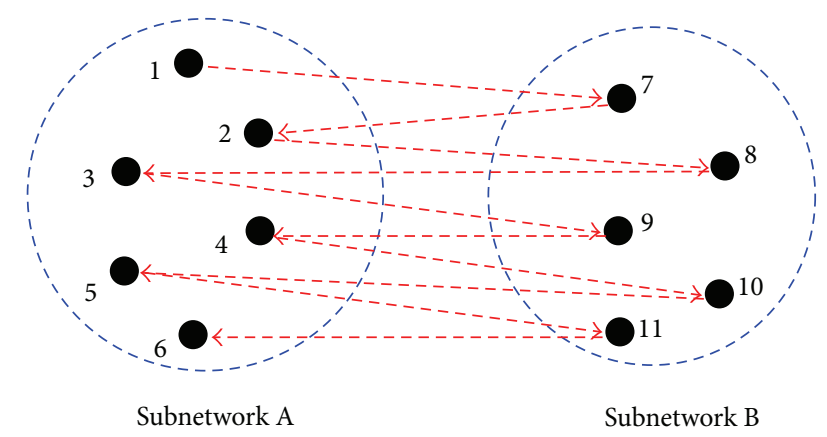

FIGURE 3: An all-negative bipartite network $\overline{\mathscr{G}}^{s}$.

assumed in Theorem 2, thus the two competitive subgroups finally reach a free bipartite consensus. In fact, the final consensus state is given by $\mathrm{c}=1.5$.

Example 9. In this example, we consider a homogenous coopetition network $\mathscr{G}^{s}$, which has all-negative edges and is illustrated in Figure 3. The coopetition network $\mathscr{G}^{S}$ is a bipartite network as well. The node 1 is still a root node of the spanning tree in $\mathscr{G}^{s}$. In order to investigate the collective dynamics on $\mathscr{G}^{s}$, the initial collective state is taken by $x(0)=$ $\operatorname{col}(1,-3,5,2,-1,4,-2,-1,3,1,-1)$.

From Figure 4, it can be found that all agents in the two competitive subnetworks $A$ and $B$ approach two final states 1 and -1 , respectively. Thus, the free bipartite consensus is still reached for the multiagent system with the control (10), even though the coopetition network is not structurally balanced.

Example 10. In order to coordinate two competitive subgroups, an effective method is to introduce one mediator, which is called a virtual leader in this paper. An interaction relationship for two competitive subgroups and a virtual leader is shown in Figure 5. Obviously, the coopetition network $\mathscr{G}^{s}$, which only involves the two competitive subnetworks, is a subgraph of the leader-involved coopetition network $\overline{\mathscr{G}}^{s}$. It is noted that the leader, labelled by 0 , is the root node of the coopetition network. The eigenvalues of the matrix $H^{s}=L^{s}+B$ are, respectively, $1,0.1981,0.382,1.555,3.247,2.618,1,3,1,1,1$, which agrees with Lemma 5.

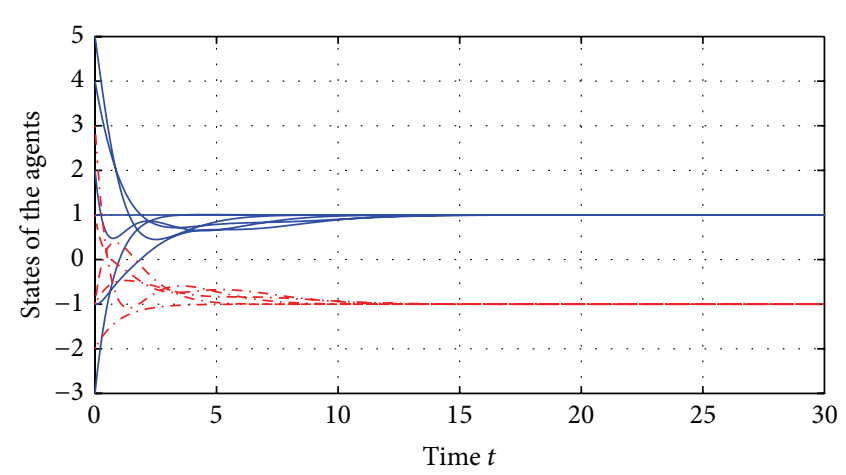

FIgURE 4: State evolution of agents on the bipartite coopetition network $\mathscr{G}^{s}$.

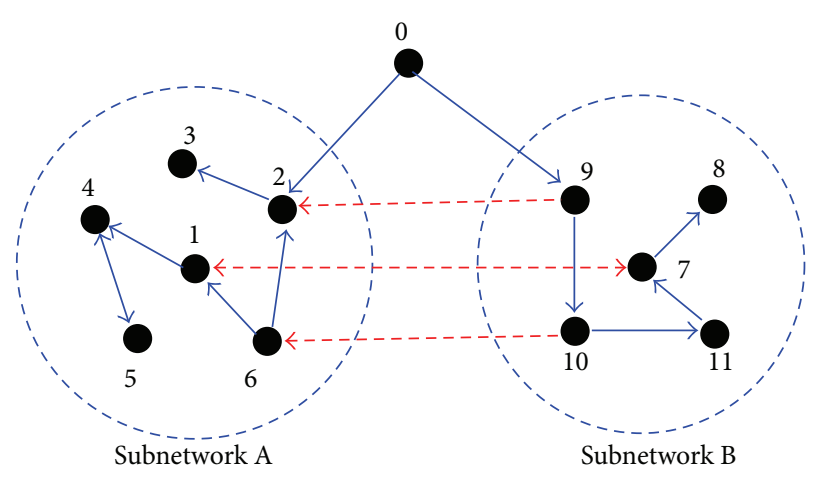

FIgURE 5: A leader-involved coopetition network $\overline{\mathscr{G}}^{s}$.

Suppose that the initial states of the two competitive subgroups are identical as those in Example 8. The input $u_{0}(t)$ of the exogenous system is described by $u_{0}(t)=0.05 \sin (t)$ and the initial state is given by $x_{0}(0)=0$. Then the state evolutions of the agents belonging to two competitive subnetworks $A$ and $B$ are illustrated in Figure 6. It is found that the agents belonging to subnetwork $A$ are approaching the state $x_{0}(t)$ of the virtual leader while the opponents are close to the reverse state $-x_{0}(t)$. Figure 6 shows that the leader can intervene in the two competitive subgroups effectively and thus guarantee the two subgroups to reach bipartite consensus with respect to the state of the leader.

\section{Conclusion}

In this paper, a bipartite consensus problem was formulated for a group of agents on coopetition networks. A free bipartite consensus control and an interventional bipartite consensus control were, respectively, proposed to ensure the realization of bipartite consensus of multiagent systems with/without exogenous influence. Under a weak connectivity assumption that the coopetition network associated with the multiagent system has a spanning tree, some sufficient conditions were obtained to describe cases in which the free bipartite consensus or the interventional bipartite consensus can be achieved. The main results showed that bipartite consensus can be achieved for bipartite networks or structurally balanced networks. Some simulations were provided to demonstrate 


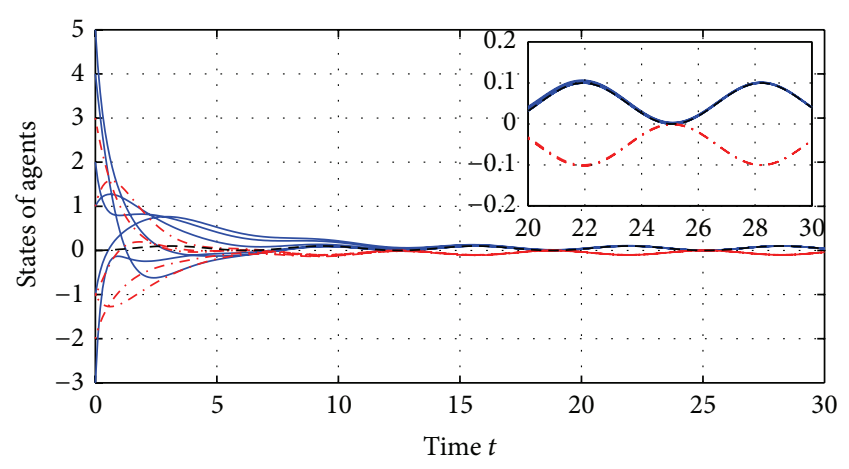

FIGURE 6: State evolution of agents on the structurally balanced coopetition network $\mathscr{G}^{s}$.

the bipartite consensus formation of multiagent systems on coopetition networks.

The results in this paper elaborate the modeling and analysis for collective dynamics over coopetition networks, which widely exist in many fields, such as opinion formation in social systems, coopetitive games in economic systems, predator-prey scenarios for animals, and antisynchronization phenomenon in chemistry and physics. Future work will focus on the case of coexisting competition/cooperation between a pair of agents and the modeling of a more general coopetition network. Some notations from overlay networks and differential game theory may provide a potential solution when a complex coopetition network can be divided into two layers of virtual signed networks and the agents are friends and opponents at the same time.

\section{Conflict of Interests}

The author declares that there is no conflict of interests regarding the publication of this paper.

\section{Acknowledgments}

This work was supported in part by the National Natural Science Foundation of China under Grant 61104104, the Program for New Century Excellent Talents in University under Grant NCET-13-0091, and the Scientific Research Foundation for the Returned Overseas Chinese Scholars, State Education Ministry of China.

\section{References}

[1] J. H. Holland, Emergence: From Chaos to Order, Helix Books, Addison-Wesley, Reading, Mass, USA, 1998.

[2] M. Mitchell and M. Newman, "Complex systems theory and evolution," in Encyclopedia of Evolution, M. Pagel, Ed., Oxford University Press, New York, NY, USA, 2002.

[3] E. Bonabeau, M. Dorigo, and G. Theraulaz, Swarm Intelligence: From Natural to Artificial System, Oxford University Press, New York, NY, USA, 1999.

[4] C. Castellano, S. Fortunato, and V. Loreto, "Statistical physics of social dynamics," Reviews of Modern Physics, vol. 81, no. 2, pp. 591-646, 2009.
[5] M. H. Degroot, "Reaching a consensus," Journal of the American Statistical Association, vol. 69, no. 345, pp. 118-121, 1974.

[6] R. E. Mirollo and S. H. Strogatz, "Synchronization of pulsecoupled biological oscillators," SIAM Journal on Applied Mathematics, vol. 50, no. 6, pp. 1645-1662, 1990.

[7] Y. Yang, D. V. Dimarogonas, and X. Hu, "Opinion consensus of modified Hegselmann-Krause models," Automatica, vol. 50, no. 2, pp. 622-627, 2014.

[8] Z. Néda, E. Ravasz, Y. Brechet, T. Vicsek, and A.-L. Barabási, "The sound of many hands clapping," Nature, vol. 403, pp. 849$850,2000$.

[9] T. Vicsek, A. Czirk, E. Ben-Jacob, I. Cohen, and O. Shochet, "Novel type of phase transition in a system of self-driven particles," Physical Review Letters, vol. 75, no. 6, pp. 1226-1229, 1995.

[10] L. M. Pecora and T. L. Carroll, "Synchronization in chaotic systems," Physical Review Letters, vol. 64, no. 8, pp. 821-824, 1990.

[11] Z. Li, Z. Duan, G. Chen, and L. Huang, "Consensus of multiagent systems and synchronization of complex networks: a unified viewpoint," IEEE Transactions on Circuits and Systems I: Regular Papers, vol. 57, no. 1, pp. 213-224, 2010.

[12] Y. Tang and W. K. Wong, "Distributed synchronization of coupled neural networks via randomly occurring control," IEEE Transactions on Neural Networks and Learning Systems, vol. 24, no. 3, pp. 435-447, 2013.

[13] Y. Cao, W. Yu, W. Ren, and G. Chen, "An overview of recent progress in the study of distributed multi-agent coordination," IEEE Transactions on Industrial Informatics, vol. 9, no. 1, pp. 427-438, 2013.

[14] W. Yu, G. Chen, J. Lü, and J. Kurths, "Synchronization via pinning control on general complex networks," SIAM Journal on Control and Optimization, vol. 51, no. 2, pp. 1395-1416, 2013.

[15] W. Ren and R. W. Beard, Distributed Consensus in Multi-Vehicle Cooperative Control: Theory and Applications, Springer, Berlin, Germany, 2008.

[16] Z. Lin, B. Francis, and M. Maggiore, "Necessary and sufficient graphical conditions for formation control of unicycles," IEEE Transactions on Automatic Control, vol. 50, no. 1, pp. 121-127, 2005.

[17] J. Hu and Y. Hong, "Leader-following coordination of multiagent systems with coupling time delays," Physica A: Statistical Mechanics and Its Applications, vol. 374, no. 2, pp. 853-863, 2007.

[18] G. Wen, Z. Li, Z. Duan, and G. Chen, "Distributed consensus control for linear multi-agent systems with discontinuous observations," International Journal of Control, vol. 86, no. 1, pp. 95-106, 2013.

[19] W. Riker, The Theory of Political Coalitions, Yale University Press, New Haven, Conn, USA, 1962.

[20] A. Ware, The Dynamics of Two-Party Politics: Party Structures and the Management of Competition, Comparative Politics, Oxford University Press, Oxford, UK, 2009.

[21] C.-M. Kim, S. Rim, W.-H. Kye, J.-W. Ryu, and Y.-J. Park, "Antisynchronization of chaotic oscillators," Physics Letters A, vol. 320, no. 1, pp. 39-46, 2003.

[22] J. Hu, S. Chen, and L. Chen, "Adaptive control for antisynchronization of Chua's chaotic system," Physics Letters A, vol. 339, no. 6, pp. 455-460, 2005.

[23] E. Ising, "Beitrag zur theorie des ferromagnetismus," Zeitschrift für Physik, vol. 31, no. 1, pp. 253-258, 1925. 
[24] F. Harary, "On the notion of balance of a signed graph," The Michigan Mathematical Journal, vol. 2, no. 2, pp. 143-146, 1953.

[25] C. Altafini, "Dynamics of opinion forming in structurally balanced social networks," PLoS ONE, vol. 7, no. 6, Article ID e38135, 2012.

[26] C. Altafini, "Consensus problems on networks with antagonistic interactions," IEEE Transactions on Automatic Control, vol. 58, no. 4, pp. 935-946, 2013.

[27] J. Hu and W. X. Zheng, "Emergent collective behaviors on coopetition networks," Physics Letters A, vol. 378, no. 26-27, pp. 17871796, 2014.

[28] S. Wasserman and K. Faust, Social Network Analysis: Methods and Applications, Cambridge University Press, Cambridge, UK, 1994.

[29] L. M. Pecora and T. L. Carroll, "Master stability functions for synchronized coupled systems," Physical Review Letters, vol. 80, no. 10, pp. 2109-2112, 1998. 


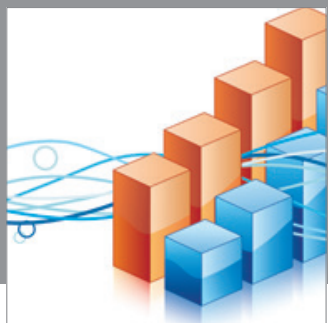

Advances in

Operations Research

mansans

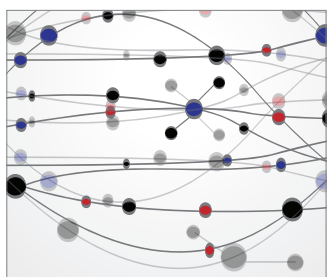

The Scientific World Journal
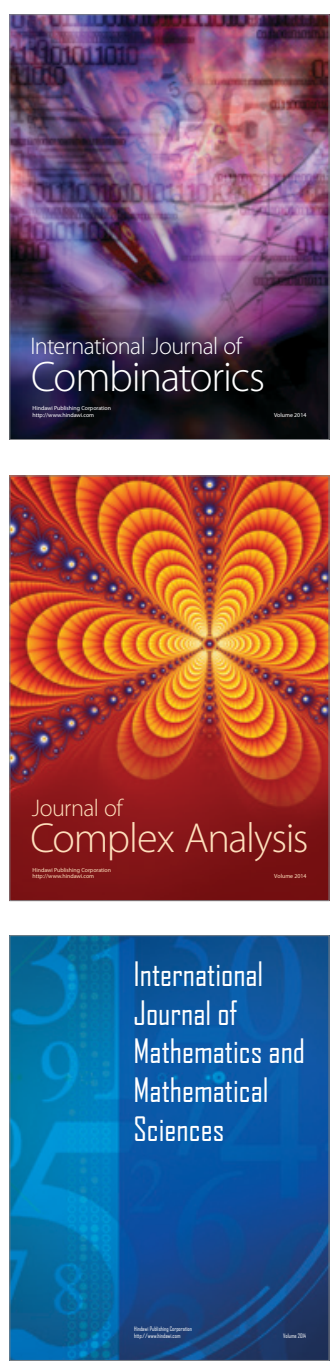
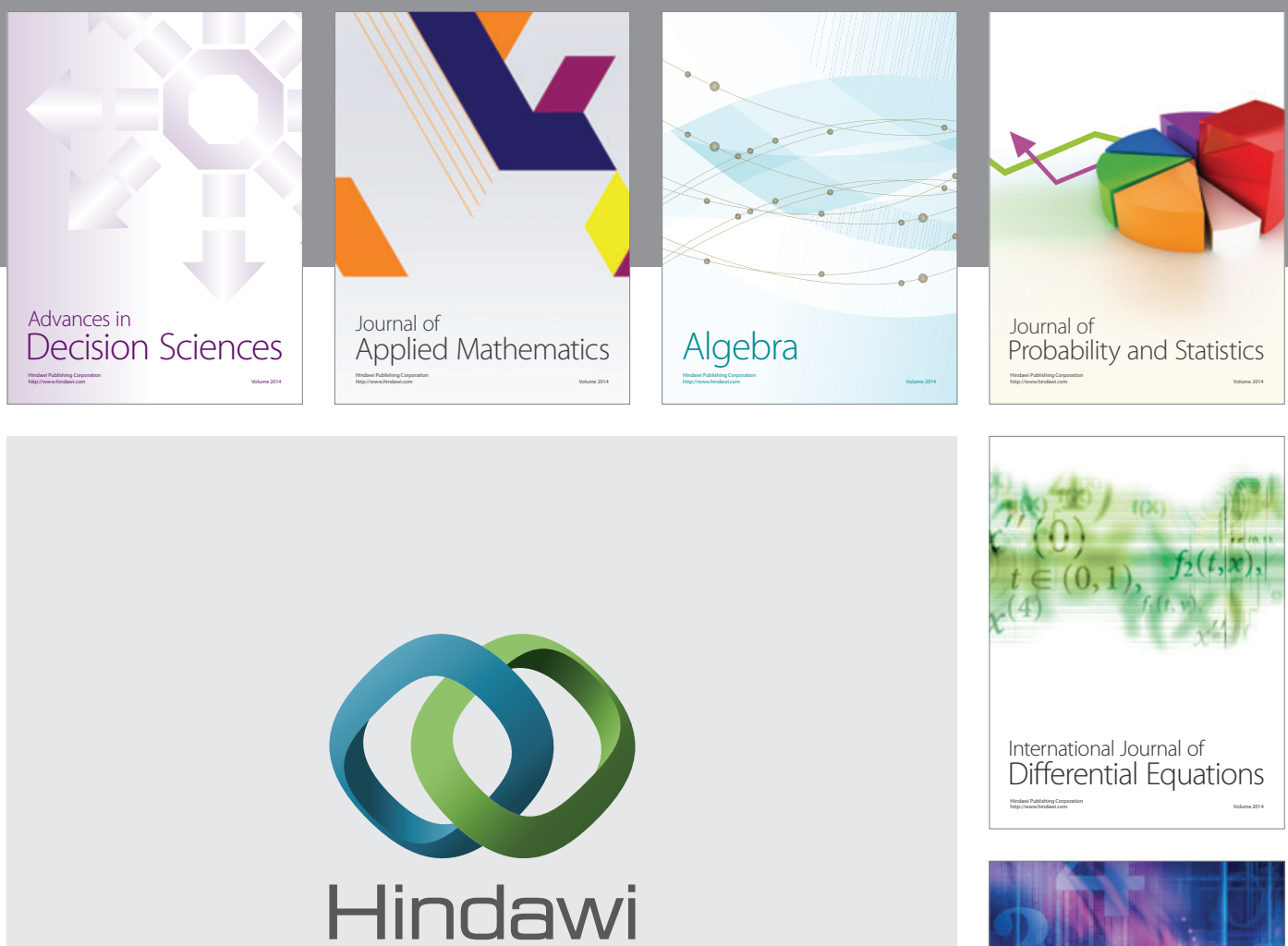

Submit your manuscripts at http://www.hindawi.com
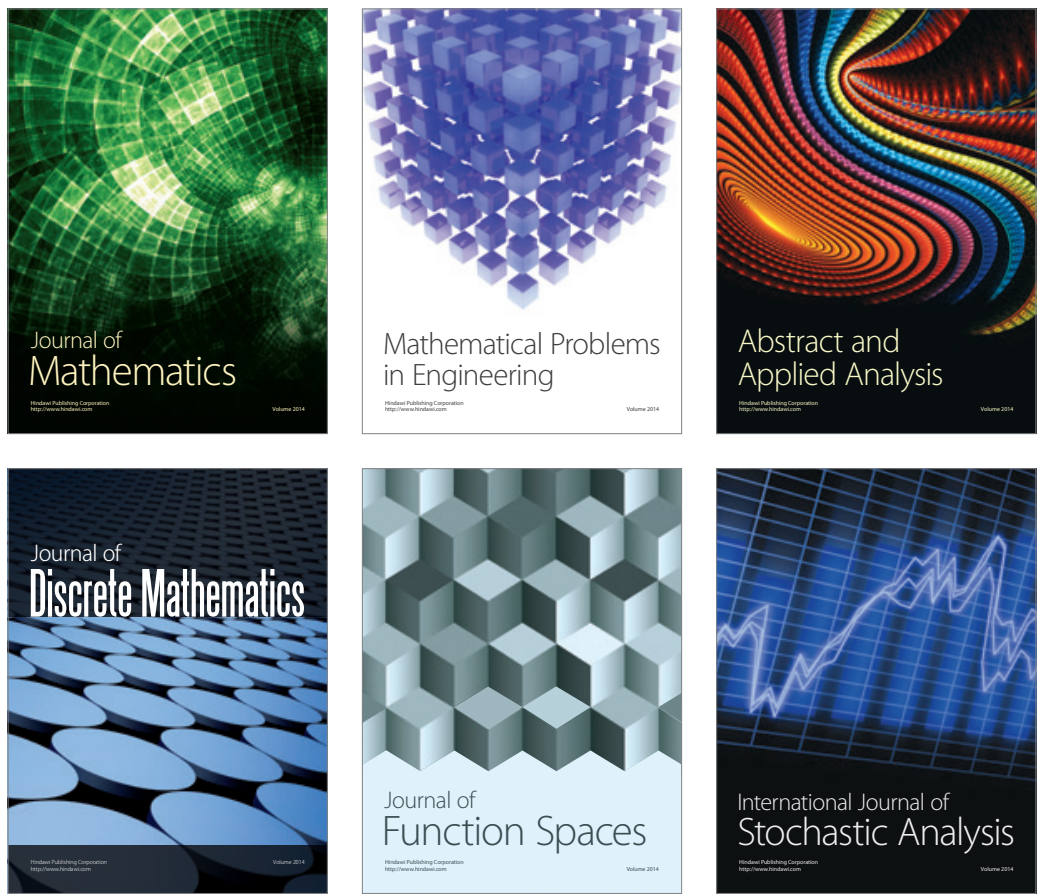

Journal of

Function Spaces

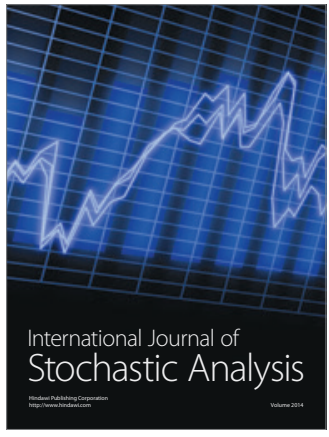

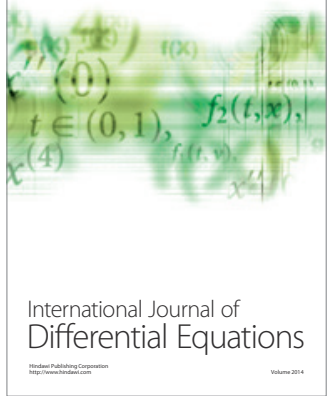
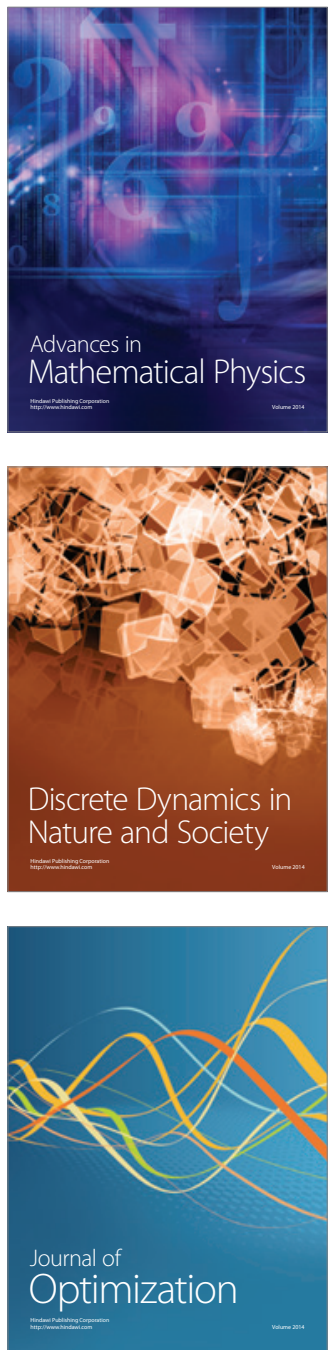